\title{
A sphere packing model for shear bands in dense soils
}

\author{
Calixtro Yanqui ${ }^{{ }^{*}}$ \\ ${ }^{1}$ Civil Engineering Department, National University of Saint Agustine. Arequipa, Peru
}

\begin{abstract}
The rhombic sphere packing can be used to model the biaxial test on granular soils in a very simple way. According to the angle of assemblage, the packing is dilatant or contractive. Correspondingly, overall stresses are transmitted as chains of forces or oblique forces of contact. The connection of the soil stress-strain behaviour and the packing void ratio is achieved by mapping both of the plots. The mapping shows that dense soils are dilatant and loose soils are contractive, separated by the critical state. It also shows that the bifurcation point and the peak strength are features only of dense soils. The band of strain localization is analysed in the elastic regime, and its inclination is found maximizing the intensity of the mobilized stress ratio. The stresses within the shear band are obtained by assuming a partially coaxial packing rotated to reach the full plastic state. The equilibrium of the overall stress at the line of discontinuity reveals a relationship between the peak friction angle and the coefficient of lateral pressure at rest. As long as these parameters are obtained independently of each other, they allow the validation of the theory.
\end{abstract}

\section{Introduction}

A shear band is the final effect of the location of deformation in a solid body. In particular, dense granular soils have been characterized by exhibiting this type of failure. Various authors have carried out experiments using increasingly sophisticated methods, such as high-speed photography, microscopic thin sections, X-rays, and Digital Image Correlation, among others [1]. For instance, the biaxial compression tests carried out in two sands by Oda and Kazama [2] have yielded the following conclusions: (a) the shear bands are not necessarily straight lines, (b) the pores in the shear band are larger than the pores in the loosest state, (c) the orientation of the grains changes at the edge or line of discontinuity, (d) the grains rotate, (e) the thickness of the shear band is between 7 to 10 times the size of the grain (f) the grains of the shear band form columns parallel to the direction of the major principal stress. The explanation of some of these characteristics is fundamentally based on two continuum theories: the mechanics of fracture, and the theory of bifurcation [3]. But the localization of the deformation is a phenomenon whose understanding cannot be achieved without taking into account the granular nature of the soil [4]. As a matter of fact, several authors have found that the assemblies of spheres used in the Distinct Element Method give results that are consistent with the experiments $[5,6]$. But an analytical and comprehensive theory of the mechanics of shear bands is still in development.

In the present work, a packing of frictionless spheres, ordered in a rhombic Bravais lattice, is proposed to model a granular soil subjected to a biaxial compression test. Likewise, the assembly of grains within the shear band is modeled by a rhombic packing rotated until the limiting equilibrium is fulfilled.

\section{Rhombic sphere packing}

Although the theory is valid for any packing, for the sake of simplicity, the rhombic sphere packing of rectangular base is chosen because it is very simple and can be applied to the description of the shear bands in the biaxial compression test for granular soils. The first step is to obtain the relationship between the void ratio $e$ and the assemblage angle, $\theta$, which represents the structure of a two-dimensional packing (Fig. 1). That is:

$$
e=\frac{6}{\pi} \sin 2 \theta-1
$$

This formula can be interpreted in two ways. (a) It describes the physical state of a soil assembly. For this purpose, it should be noted that the domain of the angle of assemblage is: $30^{\circ} \leq \theta \leq 60^{\circ}$. Both of this limits correspond to the densest state of the packing. The angle $\theta=45^{\circ}$ is a mathematical root and defines the loosest state. Therefore, function (1) has two branches, exhibiting the same void ratio for two values of the assemblage angle. (b) It serves to analysis the kinematics of the strain of a packing. Indeed, the packing undergoes a vertical strain due to the indentation of the sphere of the upper layer into the two spheres of the lower layer. The tangential displacement of the spheres in contact shows that the packing is dilatant in the interval $30^{\circ} \leq \theta \leq 45^{\circ}$, and, contractive in the interval $45^{\circ} \leq \theta \leq 60^{\circ}$. In other words, if the angle between the tangent and the line of assemblage is

* Corresponding author: cyanqui@ unsa.edu.pe

A video is available at https://doi.org/10.48448/dc68-ak08 
clockwise, the packing is dilatant, if not, the packing is contractive. This property has been called by several authors duality of packings [7].
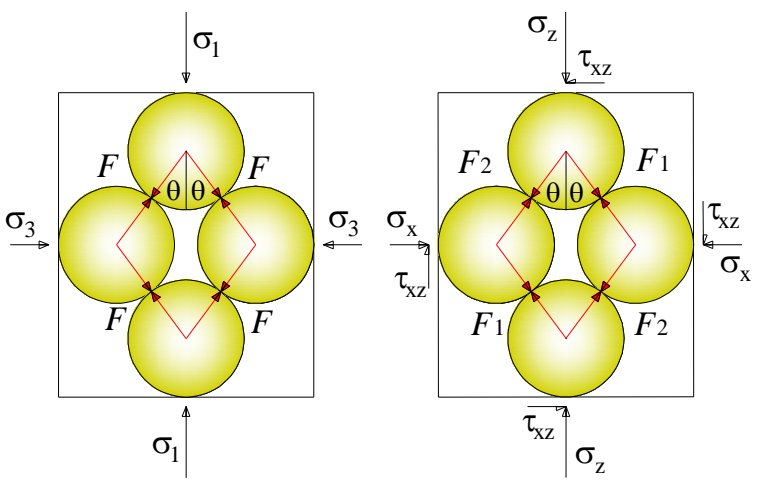

Fig. 1. Rhombic packing of spheres and forces of contact induced by overall stresses in: a) coaxial packing, and b) partially coaxial packing.

\section{Chains of forces}

In a coaxial packing, the axes of the principal stresses coincide with the rhombus axes. The static equilibrium of the overall principal stresses with the chains of contact forces yields: $F=\sigma_{1} S /(2 \cos \theta)=\sigma_{3} S \cot \theta /(2 \sin \theta)$, where $S$ is the width of the element, and $S \cot \theta$, its height. Thus, the relationship between the lateral stress, $\sigma_{3}$, and the axial principal stress, $\sigma_{1}$ is given by (Fig. 1a):

$$
\frac{\sigma_{3}}{\sigma_{1}}=\tan ^{2} \theta
$$

Equation (2) is valid only for $30^{\circ} \leq \theta \leq 45^{\circ}$. In the interval $45^{\circ} \leq \theta \leq 60^{\circ}$, the axial stress, $\sigma_{1}$, is smaller than the lateral stress, $\sigma_{3}$. This condition is against the requirement for the biaxial compressional test. The unique solution to the problem is the transmission of the overall stresses in the form of oblique contact forces, without actually forming force chains. If $\delta$ is the angle of obliquity, the equilibrium of the external and internal forces yields: $F=\sigma_{1} S /[2 \cos (\theta-\delta)]=\sigma_{3} S \cot \theta /[(2 \sin (\theta-\delta)]$, and the principal stress ratio is found to be:

$$
\frac{\sigma_{3}}{\sigma_{1}}=\tan \theta \tan (\theta-\delta)
$$

But this equation can also be used in the interval $30^{\circ} \leq \theta \leq 45^{\circ}$ when the force obliquity is important, as did Rowe [8] for the stress-dilatancy theory. In both cases, the angle $\delta$ becomes the friction angle at the ultimate state. In conclusion, chains of forces are only formed in dilatant packings, while shear forces determine the behavior of contractive packings. Among others, Amirrahmat et al. [9] have experimentally quantified these features using Synchrotron Microcomputed Tomography images.

A packing is said to be partially coaxial if the axes of the rhombus coincide with the direction of the normal stresses in an element submitted to the overall stresses $\sigma_{\mathrm{x}}, \sigma_{\mathrm{z}}$, and $\tau_{\mathrm{zx}}$ (Fig. 1b). Since the spheres are assumed to be frictionless, stresses are transmitted by asymmetric chains of normal contact forces $F_{1}$ and $F_{2}$. For instance, $F_{1}=S\left(\sigma_{\mathrm{z}} \sin \theta-\tau_{\mathrm{xz}} \cos \theta\right) / \sin 2 \theta=S\left(\sigma_{\mathrm{x}} \cos \theta-\tau_{\mathrm{xz}} \sin \theta\right) /\left(2 \sin ^{2} \theta\right)$. This identity leads to the stress ratio $\sigma_{x} / \sigma_{z}=\tan ^{2} \theta$, whose meaning is more general than equation (2). The solution of the system of three equations, formed by $\sigma_{x} / \sigma_{z}=\tan ^{2} \theta$ and the two differential equations of equilibrium, is a hyperbolic differential equation. Thus, this equation is considered by some authors as the constitutive law for two-dimensional granular matter [10, 11].

\section{Mapping of plots}

Curves OPC and OC in diagram $2 \mathrm{a}$ are standard features of the strain response during the biaxial compressional test on granular soils. They show that, for a dense assembly of grains, the deviatoric stress, $\sigma_{d}$, reaches a maximum value, at point $\mathrm{P}$, and then decreases asymptotically to attain a constant value, at point C. For a loose assembly of grains, the deviatoric stress increases monotonically until arriving to the same constant value at point $\mathrm{C}$, which is called the critical state of the soil. Since the void ratio, $e$, is a function of the linear strain, $\varepsilon$, the diagram 2 a may be mapped into the diagram $2 b$, to transform the stress-strain curves, for dense and loose states, into a two-valued asymmetric single curve. Diagram $2 b$ is very important because it establishes that a dense soil is dilatant, and loose soil is contractive, with a common value at point $\mathrm{C}[12]$.

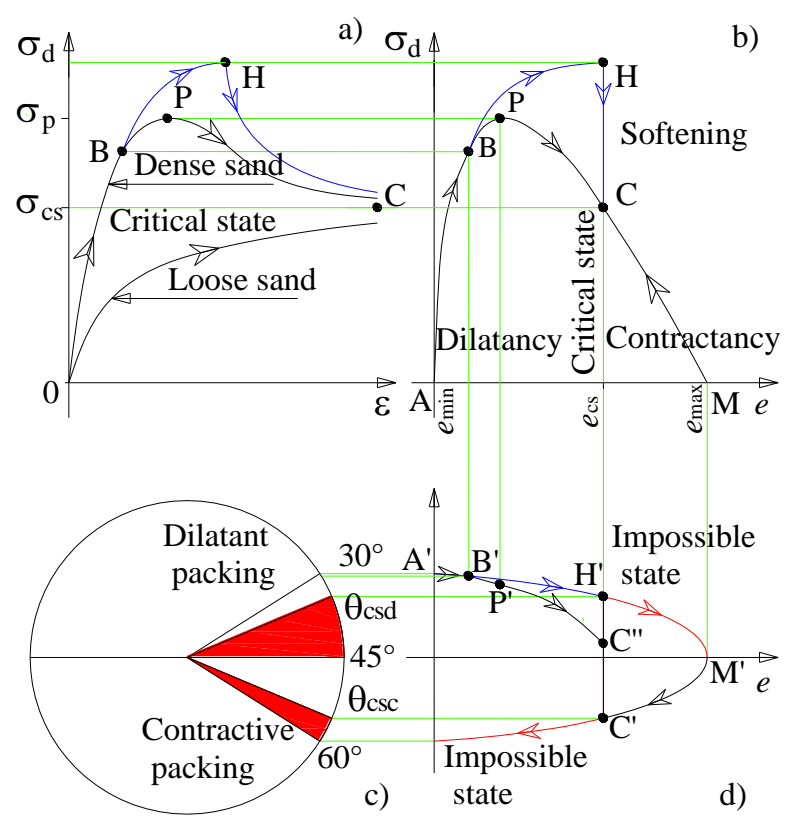

Fig. 2. Mapping of plots. a) Experimental stress-strain curves of dense and loose sands, b) Casagrande's plot [12], c) Angle of assemblage for the sphere points of contact, and d) Plot of equation (1). Black lines refer to dense soil with strain localization, and blue lines, to soil with homogeneous strain.

Likewise, the points of contact of a sphere, given by the angle $\theta$ in the figure $2 \mathrm{c}$, can be plotted against the void ratio according to the equation (1). The curve is a canonical ellipse, and consequently has two branches: 
one for the dilatant packing and another for the contractive packing. The most important property of these plots is the connection of the sphere packing theory with the overall mechanical behavior of granular soils. As the curves are vector trajectories, the mapping of diagram $2 \mathrm{c}$ onto diagram $2 \mathrm{~d}$ defines four intervals separated by the critical state C: the A'B'H' interval, for dense packings, the $\mathrm{M}^{\prime} \mathrm{C}^{\prime}$ interval, for loose packings, and two alternating impossible intervals. Accordingly, each possible branch has its own critical state, but with the same void ratio. The critical state of an assembly of grains is not related to the maximum void ratio, as erroneously is assumed in soil mechanics.

\section{Bifurcation and location}

As a consequence of the stress concentration at a rigid boundary or of some internal heterogeneity, the strain localization begins. In these places, the spheres are prone to rotate and develop local lines of discontinuity, because rotational friction is much smaller than sliding friction. This event marks a change in the slope of the stress-strain curve, from a point of bifurcation B [1]. The deviatoric stress diminishes, so does the peak resistance. As the deviatoric stress is increased, the number of these singular spheres increases, forming local lines where they act like bearings, during the strain hardening phase [9]. At the end of the process, and according to the initial density of the packing, two parallel lines of discontinuity grow and prevail, enclosing a zone in which the axes of the packings are rotated, called the shear band (Fig. 3a). Accordingly, the specimen comprises two elastic zones and a thin plastic zone.

The shear band process may be interpreted at the light of the plots mapping. For a dense homogeneous assembly of grains, the stress-void ratio response is given by the OBHC curve, whose mapping on A'B' $\mathrm{H}^{\prime}$ curve reveals a critical state of the packing model at point H'. Likewise, the M'C' curve has a critical state at point C', for a loose packing, which makes an angle of $90^{\circ}$ with the former. Thus, the line $\mathrm{H}^{\prime} \mathrm{C}^{\prime}$ is the geometrical place of the critical state of packings rotated to an angle between $0^{\circ}$ and $90^{\circ}$. But, to reach the corresponding point, the mechanical response of the rotated packing must change to the A'B'P'C', curve (Fig. 2c). The mapping of this curve in the plot 2a gives the stress-strain curve OBPC for a specimen affected by the strain localization.

\section{Stresses in the shear band}

As an elemental approach, it may be chosen a partially coaxial packing to describe the mechanical behavior of the shear band. Since the axes of the packings inside the shear band do not coincide with the axes of the specimen (Fig. 3c), Cartesian coordinates, whose z-axis has the direction of the line of failure, are taken as the frame of reference. In this system of coordinates, the overall stresses components acting on the lines parallel to the line of discontinuity must satisfy the Coulomb's law, $\tau_{\mathrm{zx}}=\sigma_{\mathrm{x}} \tan \varphi$. The angle $\varphi$ is the peak friction angle given by the OBPC curve after the bifurcation point in diagram 2a. As has been shown before, in a partially coaxial packing, the normal stresses are related by the formula: $\sigma_{\mathrm{x}}=\sigma_{\mathrm{z}} \tan ^{2} \theta$. In addition, as the shear band is a full plastic zone, the stresses must fulfill the Mohr-Coulomb law in the Cartesian form:

$$
\sqrt{\left(\sigma_{x}-\sigma_{z}\right)^{2}+4 \tau_{x z}^{2}}=\left(\sigma_{x}+\sigma_{z}\right) \sin \varphi
$$
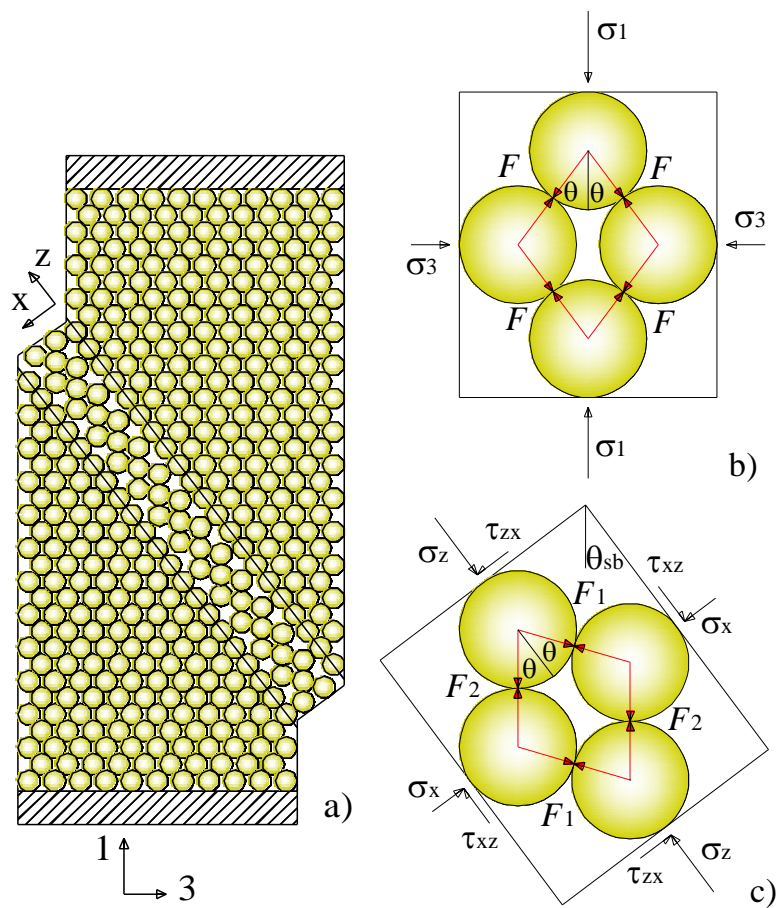

Fig. 3 Biaxial compression test: a) Assembly of ordered spheres in the elastic zones and shear band showing the higher void ratio near the lines of discontinuity, b) Packing and chains of forces in the elastic zones, and c) Packing and overall stresses at the shear band.

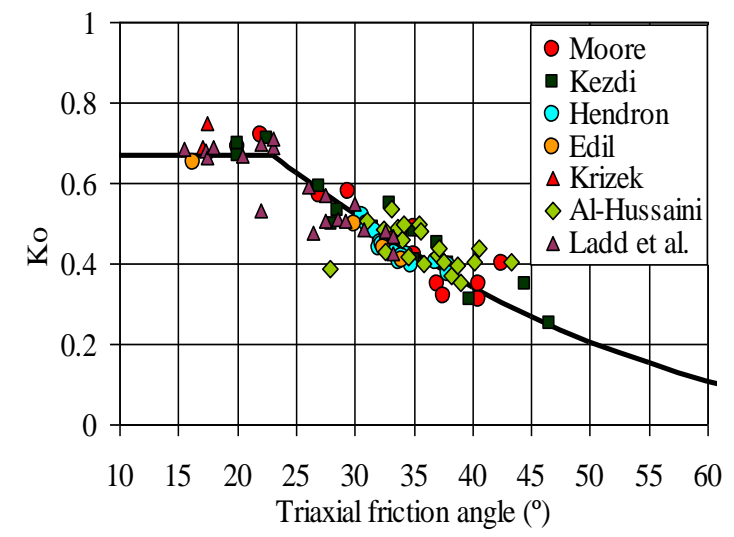

Fig. 4. Relationship between the friction angle and the coefficient of lateral pressure at rest, regarding the correction of the biaxial friction angle. Data compiled by [13].

This system of three equations has one real solution for some shear band orientation. Such a solution relates the peak friction angle of the assembly of grains, $\varphi$, to the angle of the packing of spheres, $\theta$, as follows:

$$
\tan ^{2} \theta=\frac{1}{1+2 \tan ^{2} \varphi}
$$


A relationship between the angle of assemblage of the rhombic packing and the coefficient of lateral pressure "at rest", $K_{0}$, can be established as a natural application of the theory of linear chains of forces to obtain the geostatic stresses in soils. That is: $K_{0}=\sigma_{3} / \sigma_{1}=\tan ^{2} \theta$. Consequently, as $K_{0}$ and $\varphi$ are experimental quantities, independently obtained from each other, equation (5) serves to evaluate the validity of the granular model and, particularly, the plasticity of the partially coaxial packing in the shear band. Figure 4 shows that equation (5) fits well with the experimental data reported by various authors, not only for dense soils but also for loose soils, characterized by a constant $K_{0}$.

\section{Inclination of the shear band}

The localization of deformation is an elastic feature of the assembly of grains. The evolution of the zone of intense shear can be described by the fraction $\Phi$, which relates the shear strain of the sphere packing in elastic state, $\gamma$, to the ultimate shear strain, $\gamma_{\mathrm{f}}$. For a linear elastic material, the fraction is expressed in terms of the shear stress, as: $\Phi=\tau / \tau_{\text {f. }}$ According to the Mohr-Coulomb law for granular materials, $\tau_{\mathrm{f}}=\sigma \tan \varphi$, and the fraction becomes a function of the intensity of the mobilized stress ratio: $\Phi=(\tau / \sigma) \cot \varphi$ [14]. The inclination of the shear band is obtained by maximizing this function with respect to the angle, $\alpha$, subtended by the shear band on the stress principal axis: $\partial_{\alpha}(\tau / \sigma)=0$.

Within the elastic zones, the stress components, $\sigma$ and $\tau$, can be expressed in terms of the principal stresses using the known equations of the stress transformation: $\sigma=\left(\sigma_{1}+\sigma_{3}\right) / 2-\left[\left(\sigma_{1}-\sigma_{3}\right) / 2\right] \cos 2 \alpha$, and $\tau=\left[\left(\sigma_{1}-\sigma_{3}\right) / 2\right] \sin 2 \alpha$. Given that $\sigma_{3} / \sigma_{1}=\tan ^{2} \theta$ is a constant in the elastic stage and remains in this way in the elastic zones of the sample, the inclination of the shear band is found to be:

$$
\alpha_{s b}=\theta=\arctan \frac{1}{\sqrt{1+2 \tan ^{2} \varphi}}
$$

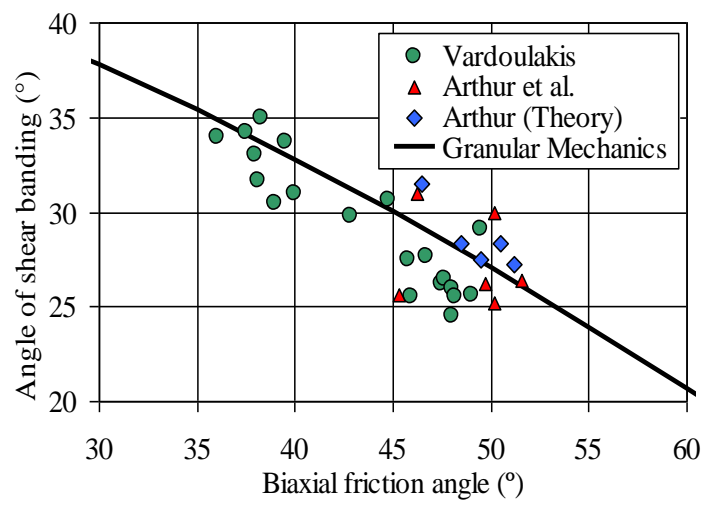

Fig. 5. Relationship between the friction angle and the inclination of shear banding in a biaxial compression test. Data compiled by [15].

From this analysis the following features are deduced: (a) the packing rotates within the shear band, (b) one of the edges of the rhombus becomes vertical, giving rise to the formation of a vertical chain of spheres (Fig. 3a), and (c) the void ratio becomes larger than the maximum void ratio, next to the lines of discontinuity. These conclusions are in agreement with the results of Oda and Kazama [2]. Likewise, figure 5 shows a good fitting of equation (6) with the experimental data.

\section{Conclusions}

The packing of ordered hard frictionless spheres is the simplest natural model for granular soil assemblies. It explains the dilatancy and the contractancy of soils, and admits the development of linear chains of forces. The connection between a soil and a packing is the mapping of plots. It explains the critical state of soils and the shear banding. Elastic zones are modeled by a coaxial packing and the plastic shear band, by a rotated partially coaxial packing. The full plastic condition within the shear band yields the relationship between the peak friction angle and the angle of packing assemblage. The maximization of the mobilized stress ratio with respect to the angle of the line of strain localization gives the inclination of the shear band. The model is validated by the experimental results reported by various authors.

\section{References}

[1] R.I. Borja, X. Song, A.L. Rechenmacher, S. Abedi, W. Wu, J. Mech. Phys. Solids 61, 219-234 (2013)

[2] M. Oda, H. Kazama, Geotechnique 48, 465-481 (1998)

[3] J.W. Rudnicki, J.B. Rice, J. Mech. Phys. Solids 23, 1501-1518 (1975)

[4] I. Vardoulakis, Localization in geomechanics, $16^{\text {th }}$ Int. Conf. on Soil Mech. Geotech. Eng. 3663-3668 (2006)

[5] K. Iwashita, M. Oda, J. Eng. Mech. ASCE 124 (3), 285-293 (1998)

[6] S. Luding, Part. Sci. Technol. 26, 33-42 (2008)

[7] S. Torquato, J. Chem. Phys. 149, 020901 (2018)

[8] P.W. Rowe, Proc. Royal Soc. London Ser. A 269 (1339), 500-527 (1962)

[9] S. Amirrahmat, A.M. Druckrey, K.A. Alshibli, R.I. Al-Raoush. J. Geotech. Geoenviron. Eng. ASCE 145 (2) (2019)

[10] R. Blumenfeld, New J. of Phys. 9, 160 (2007)

[11] A. Tordesillas, J.F. Peters, J. Shi, AIP Conf. Proc. 1542, 1218 (2013)

[12] A. Casagrande, Characteristics of cohesionless soils affecting the stability of slopes and earth fills, Contributions to Soil Mechanics, BSCE, 257-276 (1936)

[13] R. D. Holtz and Kovacs, An introduction to Geotechnical Engineering, (Prentice Hall, Englewood Cliffs, New Jersey, 1981)

[14] M. Oda, J. Konishi, Soils and Found. 14 (4), 25-38 (1974)

[15] C. Yanqui, Statics of gravitating discontinua, M.Sc. Thesis, University of South Carolina (1982) 